\title{
VARIATIONAL-LIKE INEQUALITIES FOR PSEUDOMONOTONE OPERATORS
}

\author{
ASHOK GANGULY
}

(Received 3 April 1998)

\begin{abstract}
The aim of this note is to use a fixed point theorem to prove results for
\end{abstract} variational-like inequalities for pseudomonotone operators.

2000 Mathematics Subject Classification. 47H04, 47H10.

1. Introduction. Recently, Singh et al. [10] studied pseudomonotone operators and derived interesting results in variational inequality and complementarity problems using a recent fixed point theorem of Tarafdar [13], which is equivalent to F-KKM theorem [13]. They derived a few interesting results as corollaries and gave an application in minimization problems. Earlier, Parida et al. [7] studied a variational-like inequality problem and developed a theory for the existence of its solution using Kakutani's fixed point theorem, and also established the relationship between the variational-like inequality problem and some mathematical programming problems. Further results on existence theorem for variational-like inequality problems were obtained by Wadhwa and Ganguly [14] using Tarafdar's fixed point theorem [11], which is equivalent to the KKM fixed point theorem [13].

In this note, we use Tarafdar's result [13] and prove an existence theorem for variational-like inequality problem for $g$-pseudomonotone operators and then derive some interesting results and corollaries.

We need the following definitions:

Let $E$ stand for a real locally convex Hausdorff topological vector space and $X$ a nonempty convex subset of $E$ with $E^{*} \neq\{0\}$, being the continuous dual of $E$. Let $T: X \rightarrow E^{*}$ be a nonlinear map. The mapping $T: X \rightarrow E^{*}$ is hemicontinuous if $T$ is continuous from the line segment of $X$ to the weak topology of $E^{*}$. A point $y \in X$ is said to be a solution of the variational inequality if

$$
\langle T y, x-y\rangle \geq 0 \quad \forall x \in X .
$$

Let $g$ be a continuous map, $g: X \times X \rightarrow E$. A point $y \in X$ is said to be a solution of the variational-like inequality problems if

$$
\langle T y, g(x, y)\rangle \geq 0 \quad \forall x \in X .
$$

If $g(x, y)=x-y,(1.2)$ reduces to (1.1) [7].

A map $T: X \rightarrow E^{*}$ is said to be monotone if

$$
\langle T y-T x, y-x\rangle \geq 0 \quad \forall x, y \in X
$$


Here, $(\cdot, \cdot)$ denotes the pairing between $E^{*}$ and $E$.

The map $T$ is called pseudomonotone if

$$
\langle T y, y-x\rangle \geq 0 \quad \text { whenever }\langle T x, y-x\rangle \geq 0 \forall x, y \in X .
$$

DEFINITION 1.1. A map $T: X \rightarrow E^{*}$ is said to be $g$-monotone on $X$ if

$$
\langle T x, g(y, x)\rangle+\langle T y, g(x, y)\rangle \leq 0 \quad \forall x, y \in X .
$$

For $g(y, x)=y-x$, we get the definition of monotone operators.

DEFINITION 1.2. A map $T: X \rightarrow E^{*}$ is said to be $g$-pseudomonotone if

$$
\langle T x, g(y, x)\rangle \geq 0 \quad \text { whenever }\langle T y, g(x, y)\rangle \geq 0 \forall x, y \in X .
$$

For $g(y, x)=y-x$, we get the definition of pseudomonotone operators.

We are interested in the following:

Find $x \in X$ such that

$$
\langle T x, g(y, x)\rangle+h y-h x \geq 0 \quad \forall y \in X,
$$

where $T: X \rightarrow E^{*}$ is a nonlinear mapping and $h: X \rightarrow \mathbb{R}$ is a low semi-continuous and convex functional.

We need the following fixed point theorem [13].

THEOREM 1.3. Let $X$ be a nonempty, convex subset of a Hausdorff topological vector space E. Let $F: X \rightarrow 2^{X}$ be a set-valued mapping such that

(i) for each $x \in X, f(x)$ is a nonempty, convex subset of $X$;

(ii) for each $y \in X, F^{-1}(y)=\{x \in X: y \in F(x)\}$ contains a relatively open subset $O_{y}$ of $X\left(O_{y}\right.$ may be empty for some $\left.y\right)$;

(iii) $U_{x \in X} O_{x}=X$; and

(iv) $X$ contains a nonempty subset $X_{0}$ contained in a compact convex subset $X_{1}$ of $X$ such that the set $D=\bigcap_{x \in X_{0}} O_{x}^{c}$ is compact ( $D$ may be empty and $O_{x}^{c}$ denotes the complement of $O_{x}$ in $X$ ).

Then there exists a point $x_{0} \in X$ such that $x_{0} \in F\left(x_{0}\right)$.

We make the following hypothesis.

Condition 1.4. For $X \subset E$, let $T: X \rightarrow E^{*}$ and $g: X \times X \rightarrow E$ satisfy the following:

(i) for each $x \in X, g(y, x)$ is convex $y \in X$;

(ii) $g(x, y)+g(y, z)=g(x, z)$ for all $x, y, z \in X$;

(iii) $g(x, x)=0$;

(iv) for every $x \in E^{*},\langle T x, y\rangle$ is monotone increasing in $y \in E^{*}$.

2. Main results. First, we give the following result.

LEMMA 2.1. If $X$ is a nonempty convex subset of a topological vector space $E$ and $T: X \rightarrow E^{*}$ is a g-pseudomonotone and hemicontinuous, then $x \in X$ is a solution of

$$
\langle T x, g(y, x)\rangle+h y-h x \geq 0 \quad \forall y \in X
$$


if and only if $x \in X$ is a solution of

$$
\langle T y, g(y, x)\rangle+h y-h x \geq 0 \quad \forall y \in X,
$$

where $h: X \rightarrow \mathbb{R}$ is a convex function and $g: X \times X \rightarrow E$ is such that it satisfies Condition 1.4.

Proof. Let $x \in X$ be a solution of (2.1). Then, by Condition 1.4(i), (ii) and the $g$ pseudomonotonicity of $T$, we have

$$
\langle T y, g(y, x)\rangle+h y-h x \geq 0 \quad \forall y \in X
$$

Now, assume that $x$ satisfies (2.2) and let $y \in X$ be arbitrary. Then, using Minty's technique [5],

$$
y t=(1-t) x+t y \in X \quad \forall t \in(0,1)
$$

since $X$ is convex. Hence, we have

$$
\left\langle T y_{t}, g\left(y_{t}, x\right)\right\rangle+h y_{t}-h x \geq 0
$$

So, by Condition 1.4(ii), (iii),

$$
t\left\langle T y_{t}, g(y, x)\right\rangle+t(h y-h x) \geq 0
$$

since $T$ is hemicontinuous. Letting $t \rightarrow 0$, we get

$$
\langle T x, g(y, x)\rangle+h y-h x \geq 0 .
$$

Now, we state the following result.

THEOREM 2.2. Let $X$ be a nonempty closed convex subset of a real Hausdorff topological vector space $E$ with $E^{*} \neq\{0\}$. Let $T: X \rightarrow E^{*}$ be g-pseudomonotone and hemicontinuous map such that Condition 1.4 is satisfied, and $h: X \rightarrow \mathbb{R}$ is a lower semicontinuous and convex function. Further, assume that there exists a nonempty set $X_{0}$ contained in a compact convex subset $X_{1}$ of $X$ such that the set

$$
D=\bigcap_{x \in X_{0}}\{y \in X:\langle T x, g(x, y)\rangle+h x-h y \geq 0\}
$$

is either empty or compact.

Then, there exists an $x_{0} \in X$ such that

$$
\left\langle T x_{0}, g\left(y, x_{0}\right)\right\rangle+h y-h x_{0} \geq 0 \quad \forall y \in X
$$

Proof. Suppose that, for each $y \in X$, there exists an $x \in X$ such that

$$
\langle T x, g(y, x)\rangle+h x-h y<0 .
$$


First, suppose that (2.10) does not hold. This means that there exists at least one $y_{0} \in X$ such that

$$
\left\langle T x, g\left(y_{0}, x\right)\right\rangle+h x-h y_{0} \geq 0 \quad \forall x \geq X,
$$

that is, $y_{0} \geq X$ is a solution of (2.2). Then, by Lemma 2.1, $y_{0} \in X$ is a solution of (2.1).

Next, assume that there is no solution of (2.1) under condition (2.10) given that (2.10) holds. Then, for each $x \in X$, the set

$$
F(x)=\{y \in X:\langle T x, g(y, x)\rangle+h y-h x<0\}
$$

must be nonempty. It also follows from the convexity of $h$ and by Condition 1.4 that the set $F(x)$ is convex for each $x \in X$. Thus, $F: X \rightarrow 2^{X}$ is a set-valued map with $F(x)$ nonempty and convex for each $x \in X$.

Now, for each $x \in X$,

$$
F^{-1}(x)=\{y \in X: x \in(y)\}=\{y \in X:\langle T y, g(x, y)\rangle+h x-h y<0\} .
$$

For each $x \in X$,

$$
\begin{aligned}
\left\{F^{-1}(x)\right\}^{c} & =\text { complement of } F^{-1}(x) \text { in } X \\
& =\{y \in X:\langle T y, g(x, y)\rangle+h x-h y \geq 0\} \\
& \subset\{y \in X:\langle T x, g(x, y)\rangle+h x-h y \geq 0\}
\end{aligned}
$$

by the $g$-pseudomonotonicity of $T=G(x)$.

Again, using Condition 1.4 and the convexity of $h$, we can show that $G(x)$ is convex for each $x \in X$. Since $g$ is continuous and $h$ is lower semi-continuous, $G(x)$ is a relatively closed subset of $X$.

Hence, for each $x \in X$,

$$
F^{-1}(x) \supset[G(x)]^{c}=0_{x} \quad \text { is a relatively open subset of } X .
$$

Now, by condition (2.10), we can easily see that $\bigcup_{x \in X} O_{x}=X$. (Indeed, if $y \in X$, by (2.10), there exists an $x \in X$ such that $y \in[G(x)]^{c}=O_{x}$. Thus, $y \in \bigcup_{x \in X} O_{x}$. Hence, $\bigcup_{x \in X} O_{x}=X$.)

Finally, $D=\bigcap_{x \in X_{0}} G(x)=\bigcap_{x \in X_{0}} O_{x}^{c}$ is compact or empty by the given condition. Hence, by Theorem 1.3, there exists an $x \in X$ such that $\langle T x, g(x, x)\rangle+h x-h x<0$, which is impossible. Hence, there is a solution in this case as well.

Here, we give a few results that are special cases of Theorem 2.2.

COROLlary 2.3. Let $T: X \rightarrow E^{*}$ be $g$-monotone and hemicontinuous, where $g$ satisfies Condition 1.4, $h: X \rightarrow \mathbb{R}$ is convex and lower semi-continuous. Further, assume that there exists a nonempty set $X_{0}$ contained in a compact convex subset $X_{1}$ of $X$ such that $D=\bigcap_{x \in X_{0}}\{y \in X:\langle T x, g(x, y)\rangle+h x-h y \geq 0\}$ is either empty or compact. Then there is an $x \in X$ satisfying (2.1).

REMARK 2.4. For $g(x, y)=x-y$, Corollary 2.3 implies Corollary 1.2 of Singh et al. [10] which, in turn, implies a well-known result of Tarafdar [12]. 
Corollary 2.5. Let $X$ be a compact convex subset of $E$ and $T: X \rightarrow E^{*}$ be $g$ pseudomonotone and hemicontinuous where $g$ satisfies Condition 1.4. Suppose that $h: X \rightarrow \mathbb{R}$ is lower semicontinuous and convex. Then there is an $x \in X$ satisfying (2.1).

REMARK 2.6. For $g(x, y)=x-y$,

(i) Corollary 2.5 implies [10, Corollary 1.3].

(ii) If we take $T=A-B$, where $A$ is a monotone map and $B$ is antimonotone and both are hemicontinuous, then we derive a result due to Siddiqui et al. [8]. Here, we need only two conditions, the lower semicontinuity, and the convexity of the function $h$.

REMARK 2.7. For $h=0$, Corollary 2.5 implies Theorem 2 and Corollary 1 of Wadhwa and Ganguly [14] which implies, respectively, Theorem 2 and Corollary of Tarafdar [11]. Tarafdar's result covered the result of Browder [1] and Theorem 1.1 of Hartman and Stampacchia [3].

Now, we prove a result similar to Theorem 2.1 of Singh et al. [9]. For $A \subset E$, $\operatorname{int}(A)$ and $\partial(A)$ denote, respectively, the interior and the boundary of $A$, while for $A, X \subset E$, $\operatorname{int}_{x}(A)$ and $\partial(A)$ denote, respectively, the relative interior and the relative boundary of $A$ in $X$. A subset of a Banach space is said to be solid if it has a nonempty interior.

THEOREM 2.8. Let $X$ be a closed convex subset of a reflexive Banach space $E$ and $T: X \rightarrow E^{*}$ a $g$-pseudomonotone and hemicontinuous mapping, $g: X \times X \rightarrow E$ satisfy Condition 1.4, and $h$ is convex and lower semicontinuous. Then the following conditions are equivalent:

(i) There exists $\bar{x} \in X$ such that $\langle T \bar{x}, g(x, \bar{x})\rangle+h x-h \bar{x} \geq 0$ for all $x \in X$, that is, $x$ is a solution of (2.1).

(ii) There exists a $u \in X$ and a constant $r>\|u\|$ such that $X\langle T(x), g(x, u)\rangle+h x-$ $h u \geq 0$ for all $x \in X$ with $\|x\|=r$.

(iii) There exists $r>0$ such that the set $\{x \in X:\|x\| \leq r\}$ is nonempty with the property that, for each $x \in X$ with $\|x\|=r$, there exists $a u \in X$ with $\|u\|<r$ and $\langle T(x), g(x, u)\rangle h x h u \geq 0$.

Proof. This can be proved following Cottle and Yao [2, Theorem 2.2] as well as Parida et al. [7, Theorem 3.4].

REMARK 2.9. For a monotone $T$ operator and $h=0$ :

(1) Theorem 2.8(i), (ii), and (iii) were obtained by Parida et al. [7].

(2) For $g(x, \bar{x})=x-\bar{x}$, Theorem 2.8(ii) and (iii) reduce to the results of Theorems 2.3 and 2.4 of Moré [6], respectively.

REMARK 2.10. For $g(x, x)=x-\bar{x}$ and $h=0$, Theorem 2.8(i), (ii), and (iii) were obtained as Theorem 2.1(i), (ii), and (iii) by Singh et al. [9] and, in Hilbert spaces, similar results were obtained by Cottle and Yao (see [1, Theorem 2.2]).

Let $H, K$ be nonempty, closed subsets of $\mathbb{R}^{n}$, then we denote, by $B_{H}(K)$, the set of $z \in K$ such that $U(z) \cap(H-K) \neq \Phi$ and, by $I_{H}(K)$, the set of $z \in K$ such that $U(z) \cap(H-K)=\Phi$, for some neighbourhood $U(z)$ of $z$. 
Finally, we present a result similar to Hirano and Takahashi [4] for unbounded subsets in $\mathbb{R}^{n}$. Before that, we present the following result of Singh et al. [9, Corollary 1.12].

COROLLARY 2.11. Let $X$ be a closed bounded convex subset of a reflexive Banach space $E$ and $T: X \rightarrow E^{*}$ a pseudomonotone and hemicontinuous mapping. Then the set of solutions of variational inequality for a point $x_{0} \in X,\left\langle T x_{0}, y-x_{0}\right\rangle \geq 0$ for all $y \in X$; $y \in x$; is a nonempty weakly compact convex subset of $X$.

THEOREM 2.12. Let $X$ be a nonempty closed convex subset of $\mathbb{R}^{n}$ and $T: X \rightarrow \mathbb{R}^{n}$ be $g$ pseudomonotone such that Condition 1.4 is satisfied; $h: X \rightarrow \mathbb{R}$ a lower semicontinuous and convex function. Then there exists a solution of (2.1) in $X$ if and only if there exists a bounded closed convex subset $K$ of $X$ such that, for each $z \in B_{x}(K)$, there exists $y \in I_{x}(K)$ such that

$$
\left\langle T z, g\left(y^{*}, z\right)\right\rangle+h z-h y \longrightarrow 0 .
$$

Proof. Using Corollary 2.11, with little modification, it can be shown that if there exists a solution of (2.1), then there exists a weakly compact convex subset $K$ of $X$ such that (2.16) is satisfied. Conversely, let $K$ be a weakly compact convex subset and there exists $x^{*} \in K$ such that

$$
\left\langle T x^{*}, g\left(x, x^{*}\right)\right\rangle \geq 0 \quad \forall x \geq K,
$$

where $T$ is a $g$-pseudomonotone operator. The rest of the proof is similar to that of Theorem 3 of Wadhwa and Ganguly [14].

ACKNOWLEDGEMENT. We are indebted to Prof S. P. Singh, Canada, for his kind help in the preparation of this note.

\section{REFERENCES}

[1] F. E. Browder, Nonlinear monotone operators and convex sets in Banach spaces, Bull. Amer. Math. Soc. 71 (1965), 780-785. MR 31\#5112. Zbl 138.39902.

[2] R. W. Cottle and J. C. Yao, Pseudo-monotone complementarity problems in Hilbert space, J. Optim. Theory Appl. 75 (1992), no. 2, 281-295. MR 93i:47098. Zbl 795.90071.

[3] P. Hartman and G. Stampacchia, On some non-linear elliptic differential-functional equations, Acta Math. 115 (1966), 271-310. MR 34\#6355. Zbl 142.38102.

[4] N. Hirano and W. Takahashi, Existence theorems on unbounded sets in Banach spaces, Proc. Amer. Math. Soc. 80 (1980), no. 4, 647-650. MR 82b:47068. Zbl 471.49011.

[5] G. J. Minty, Monotone (nonlinear) operators in Hilbert space, Duke Math. J. 29 (1962), 341-346. MR 29\#6319. Zbl 111.31202.

[6] J. J. Moré, Coercivity conditions in nonlinear complementarity problems, SIAM Rev. 16 (1974), 1-16. MR 49\#1270. Zbl 272.65041.

[7] J. Parida, M. Sahoo, and A. Kumar, A variational-like inequality problem, Bull. Austral. Math. Soc. 39 (1989), no. 2, 225-231. MR 90c:49013. Zbl 649.49007.

[8] A. H. Siddiqui, Q. H. Ansari, and K. R. Kazmi, On nonlinear variational inequalities, Indian J. Pure Appl. Math. 25 (1994), no. 9, 969-973. Zbl 817.49012.

[9] S. P. Singh, E. Tarafdar, and B. Watson, Variational inequalities for a pair of pseudomonotone functions, Far East J. Math. Sci. Special Volume, Part I (1996), 31-52. MR 98d:49011. Zbl 931.47054.

[10] _ Variational inequalities and applications, Indian J. Pure Appl. Math. 28 (1997), no. 8, 1083-1089. CMP 1470 123. Zbl 880.49009. 
[11] E. Tarafdar, On nonlinear variational inequalities, Proc. Amer. Math. Soc. 67 (1977), no. 1, 95-98. MR 57\#7267. Zbl 369.47029.

[12] _ _ Variational problems via a fixed point theorem, Indian J. Math. 28 (1986), no. 3, 229-240 (1987). MR 88j:47081. Zbl 641.49005.

[13] _ _ A fixed point theorem equivalent to the Fan-Knaster-Kuratowski-Mazurkiewicz theorem, J. Math. Anal. Appl. 128 (1987), no. 2, 475-479. MR 89a:47084. Zbl 644.47050.

[14] K. K. Wadhwa and A. Ganguly, Variational like inequality problem, Bull. Calcutta Math. Soc. 88 (1996), no. 1, 71-74. CMP 1448 983. Zbl 887.49009.

Ashok Ganguly: Department of ApPlied Mathematics, Shri G.S. InSTITUTE OF TeCHNOLOGY AND SCIENCE, 23, PARK ROAD, INDORE 452-003, INDIA

E-mail address: di rector@gsits. ernet.in 


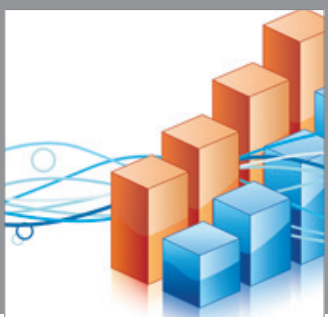

Advances in

Operations Research

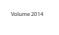

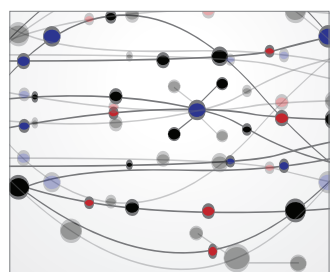

\section{The Scientific} World Journal
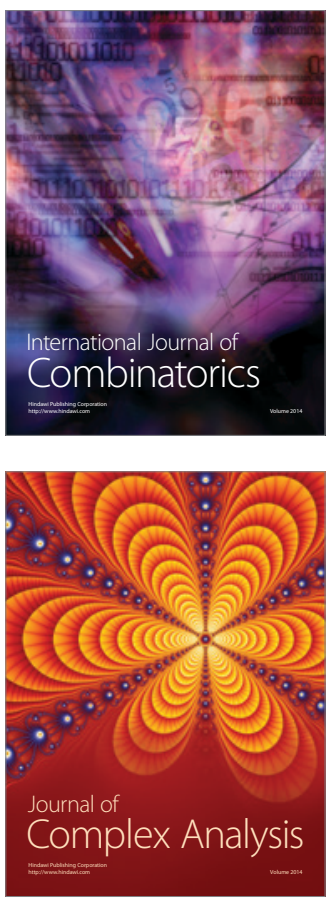

International Journal of

Mathematics and

Mathematical

Sciences
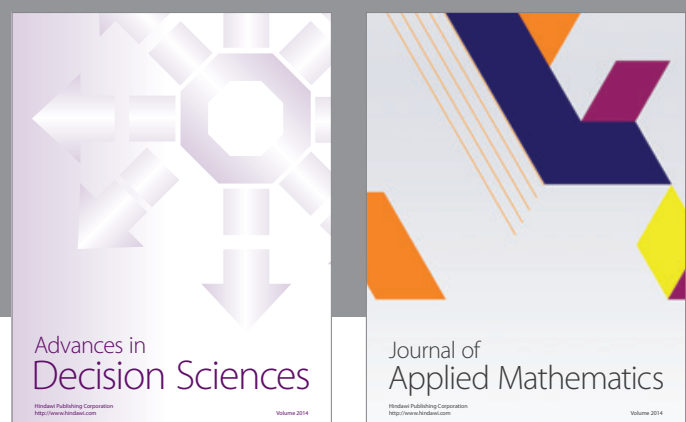

Journal of

Applied Mathematics
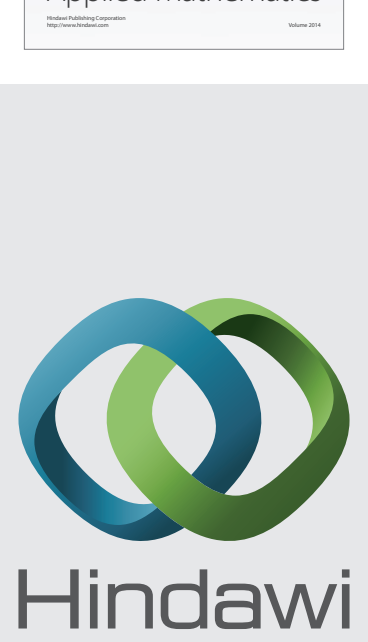

Submit your manuscripts at http://www.hindawi.com
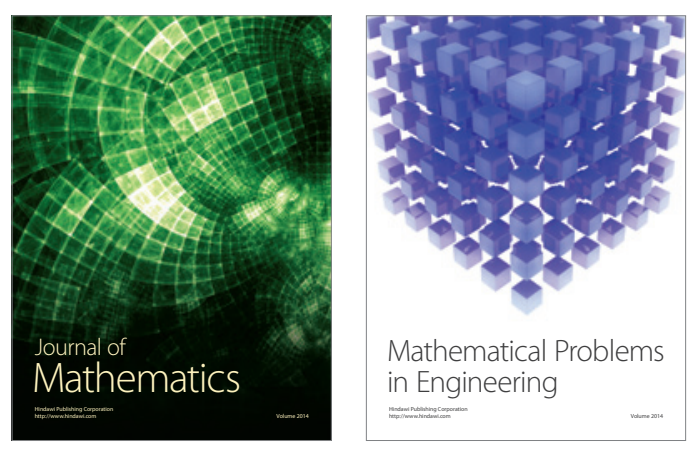

Mathematical Problems in Engineering
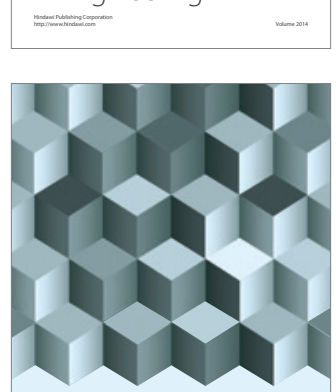

Journal of

Function Spaces
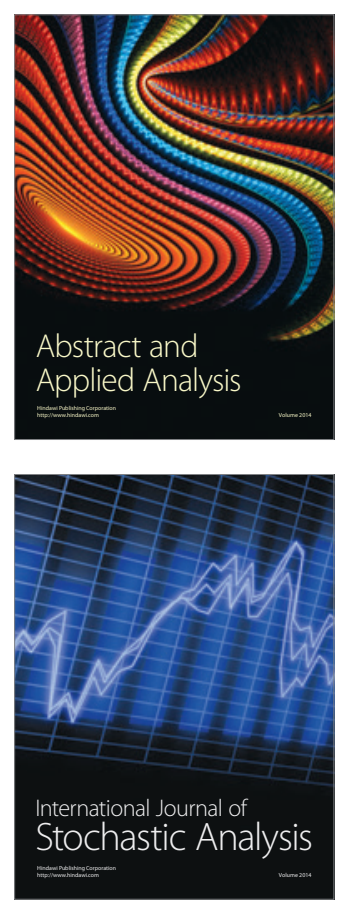

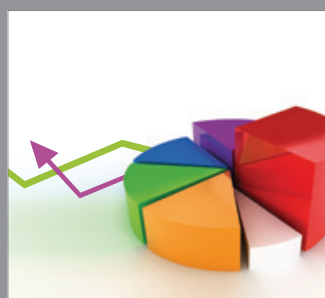

ournal of

Probability and Statistics

Promensencen
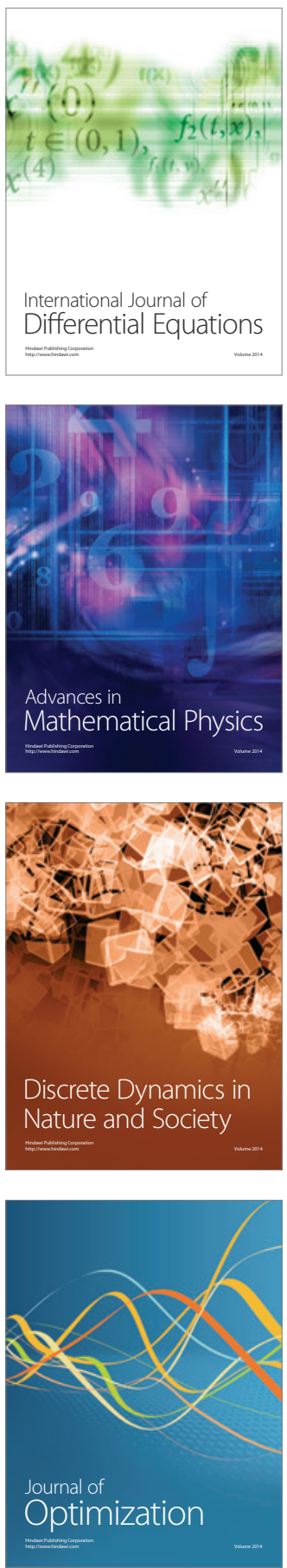